\title{
Risk minimal routes for emergency cars
}

\author{
M. Woelki, R. Nippold, M. Bonert \& S. Ruppe \\ Traffic Management, German Aerospace Center, Berlin, Germany
}

\begin{abstract}
The computation of an optimal route for a given start and destination in a static transportation network is used in many applications of private route planning. In this work we focus on route planning for emergency cars, such as, for example, police, the fire brigade and ambulance. In the case of private route planning, typical quantities to be minimized are travel time or route length. However, the idea of this paper is to minimize the risk of a travel time exceeding a certain limit. This is inspired by the fact that emergency cars have to reach the destination within a legal time. We consider mainly two approaches. The first approach takes into account relevant information to determine the weight, i.e. the desirability of certain edges of a graph during the minimization procedure. One possible risk factor to be aware of would be a suddenly jammed single-lane road on which the emergency car has no chance to make use of the benefits of the siren for instance. The same holds for full-closure situations and railroad crossings. We present a catalogue of risk factors along with an appropriate algorithm for practical route planning in emergency situations. The second one takes into account a weekly updated set of probe-vehicle data for each minute of the week along with data of current travel times. Comparing those travel-time data allows calculation of the associated risk for traveling certain edges of a route in a road network. We expect our algorithm to be a major advancement especially for destinations that lie outside the typical region travelled weekdays. In this case, automatic route planning naturally goes along with an additional gain of time.
\end{abstract}

Keywords: route planning, static transportation network, emergency situation.

\section{Introduction}

The road network is a 'critical infrastructure' whose 'failure or impairment would cause a sustained shortage of supplies, significant disruptions to public 
order or other dramatic consequences' [1]. For emergency services road network is the backbone of their mobility. Also the individual traffic, the public transport and the commercial transport requires a safe and reliable operation traffic system. But traffic management often only considers a balance between supply and demand. Potential threats and hazards and the resulting damages are not been covered. From the point of view of a critical infrastructure, traffic management must operate a risk management to evaluate the existing threads to prepare appropriate measures. Similarly, emergency services should also note risk factors for route planning in order to get not only fast, but also reliable to their destination. To address this risk evaluation in emergency services and traffic management, this paper presents several points of view of risk in this context of traffic. First, from the view of a single vehicle route planning, we present generalizations of shortest-path problems taking certain risk factors into account. This might for instance be the loss of travel time due to various reasons as traffic jam, a blockade, and narrow single-lane roads with inefficient manoeuvrability and so on. There are certain differences to private route planning with regard to the risk of exceeding a limiting travel time; since emergency cars using their siren have 'rights of way', one can assume constant loss of time when turning whether there is a traffic light or not. Even traffic jams on broader multilane roads are not as limiting for the travel time as for individual drivers since other cars have to form an emergency lane. While its formation on a large Autobahn section for example might be inefficient, it may be practicable on shorter distances as tailbacks at traffic-light crossings. Thus, risk factors might further imply the average number of lanes per kilometer, the length of the route, etc., influencing the algorithmic route choice.

Second, from the view of a traffic management center, we analyze real traffic data from floating cars in order to calculate the risk for a given route on a statistical basis.

\section{Advanced routing algorithm for emergency cars}

We consider the problem of finding a desirable route for given start and destination points on a road network. Such networks can be considered as a directed graph comprising nodes and edges so that each edge represents a road section. Edges can uniquely be classified by source node $u$ and target node $v$ as $e=(u, v)$. We consider 'static routing', i.e. the route is calculated once before the trip. Usually, static routing algorithms generalize Dijkstra's algorithm, see [2], which finds the optimal path for a graph with non-negative edge path weights. If those weights are given by length, Dijkstra gives the shortest route, while, if the weights are travel times, it provides the fastest route, see [3, 4] for an overview of the various algorithms. In general one associates a weight $T(e)$ with each edge $e$. The algorithm then finds the minimal sum of weights along a path from its source to destination node.

The interest of the present article is in assuming different risk factors as weights along the path. An example: driving on single-lane roads has the risk of being blocked; the more lanes, the easier it becomes to find a gap or even an 
emergency lane that is formed by other cars and therefore the lower is the risk. Let $n(e)$ be the number of lanes on edge $e$ and $l(e)$ its length. We are aiming to maximize the average number of lanes per kilometer:

$$
<n(e)>=\sum_{e^{\prime} \in \text { route }} l\left(e^{\prime}\right) n\left(e^{\prime}\right) / \sum_{e^{\prime} \in \operatorname{route}} l\left(e^{\prime}\right)
$$

Note that this can be achieved by an appropriate telescope sum similarly as it is done in the $\mathrm{A}^{*}$ algorithm [5]. In the same way we can find optima for other quantities; for instance the average number of lanes per second is obtained by replacing $l(e)$ by the travel time $t(e)$ and so on.

Table 1: Different risk factors as average length, travel time and number of lanes per kilometre for driving different routes at a constant amount $a$ of the speed limit.

\begin{tabular}{|l|c|c|c|c|}
\hline Route & $\begin{array}{l}\text { Extremal } \\
\text { property }\end{array}$ & length & travel time & $\begin{array}{c}<\mathbf{n} / \\
\mathbf{k m}>\end{array}$ \\
\hline $\begin{array}{l}\text { Fig. 1a) } \\
\text { dotted }\end{array}$ & Min. time & $5257 \mathrm{~m}$ & $\begin{array}{c}4: 17 \mathrm{~min} \text { for } a=1 \\
8: 00 \mathrm{~min} \text { for } a=0.535\end{array}$ & 1,688 \\
\hline $\begin{array}{l}\text { Fig. 1a) } \\
\text { black }\end{array}$ & Min. length & $4551 \mathrm{~m}$ & $\begin{array}{c}4: 39 \min \text { for } a=1 \\
8: 00 \mathrm{~min} \text { for } a=0.577\end{array}$ & 1,715 \\
\hline
\end{tabular}

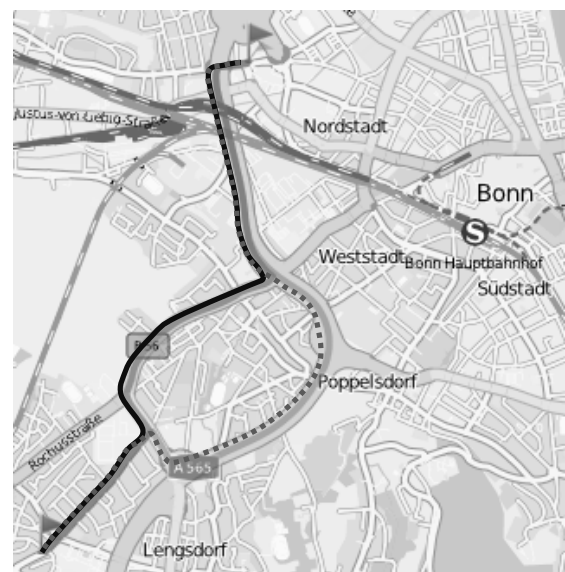

a) The dotted route is 'fastest': $T(e)=t(e)$ and travels a longer distance on the Autobahn. The black route is 'shortest': $T(e)=l(e)$.

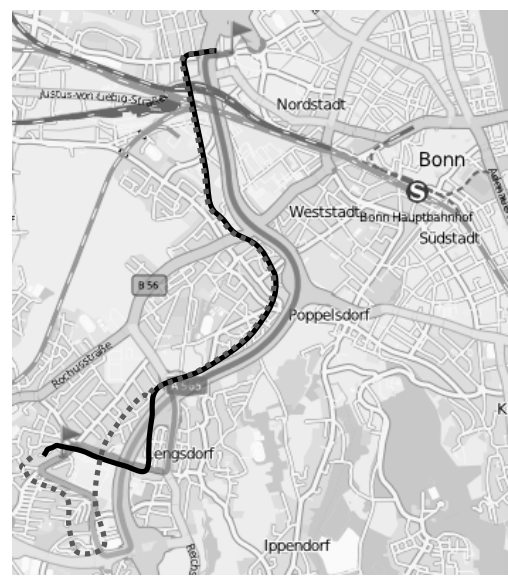

b) The dotted route is Pareto-optimal for lane number and travel time. The black route is the fastest route avoiding lowspeed edges, see text.

Figure 1: Route from fire station 1 in Bonn (north) to destination in BonnHardtberg (south).

Along with the risk factor one wants to optimize the travel time simultaneously. Obviously, this is not possible in general. However, as claimed in [6], one can determine a privileged set of paths, the set of Pareto-optimal paths. For the latter paths it is not possible to improve one of the elements 
without worsening the other. Table 1 compares essential quantities of the different routes from Figure 1. Here we assume that the emergency car travels each edge $e$ with some velocity $a$ times $v_{l i m}(e)$, with $a$ being a positive real parameter and $v_{\text {lim }}$ its speed limit.

In Bonn, emergency cars are constrained to reach their destination within 8 minutes in at least $90 \%$ of the cases of emergency. Table 1 shows that the two routes of Figure 1a) lead to the destination if the emergency car can drive at least $54 \%$ of the speed limit on the fastest route and $58 \%$ of the velocity on the shortest route. Sometimes it is necessary to take into account that emergency cars need time for turning also when making use of the siren in emergency situations. Table 2 shows a possible choice for the angle-dependent turning times. We checked that both, the fastest and the shortest route remain stable under the introduction of turning penalties $2 a$ times as large as those in table 2. In general, one might think of a more sophisticated choice of turning penalties, dependent on the turning possibilities or even taking probe-vehicle data into account $[7,8]$.

Table 2: The table gives one useful choice of weights for turning from edge $e$ ' to edge $e$ depending on the angle in between. Note that the weight can be scaled by some factor to account for different types of turning situations.

\begin{tabular}{|l|l|l|}
\hline Weight $/[\mathbf{s}]$ & Right turn: absolute angle... & Left turn: absolute angle... \\
\hline 0 & $\ldots$ smaller than $45^{\circ}$ & $\ldots$ smaller than $45^{\circ}$ \\
\hline 1 & $\ldots$ between $45^{\circ}$ and $135^{\circ}$ & \\
\hline 2 & $\ldots$ exceeding $135^{\circ}$ & $\ldots$ between $45^{\circ}$ and $135^{\circ}$ \\
\hline 3 & & $\ldots$ exceeding $135^{\circ}$ \\
\hline
\end{tabular}

Table 3: Risk factors as in Table 1 for the routes of Figure 1b) for driving different routes at a constant amount $a$ of the speed limit.

\begin{tabular}{|c|c|c|c|c|}
\hline Route & Extremal property & length & travel time & $\begin{array}{l}<n \\
k m>\end{array}$ \\
\hline $\begin{array}{l}\text { Fig. } \\
\text { 1b) } \\
\text { dotted }\end{array}$ & $\begin{array}{l}\text { Pareto-optimal for } \\
\text { max. \#lanes / km and } \\
\text { min. travel time }\end{array}$ & $6855 m$ & $\begin{array}{l}5: 00 \min \text { for } a=1 \\
8: 00 \min \text { for } a=0.625\end{array}$ & 1,900 \\
\hline $\begin{array}{l}\text { Fig. } \\
\text { 1b) } \\
\text { black }\end{array}$ & $\begin{array}{l}\text { Min. time for } \\
\text { avoiding roads with } \\
\text { speed limits lower } \\
\text { than } 50 \mathrm{~km} / \mathrm{h}\end{array}$ & $5618 m$ & $\begin{array}{l}4: 19 \min \text { for } a=1 \\
8: 00 \min \text { for } a=0.540\end{array}$ & 1,680 \\
\hline
\end{tabular}

Up to now we have assumed that emergency cars drive at a constant amount $a$ of the speed limit which is not always the case. In general, this amount will depend on the very edge, see section 4 . If possible, an emergency car might even exceed the allowed speed limit. In doing so, the driver is bound in law to make sure that he doesn't endanger other people. This is typically warranted on the Autobahn. If the emergency car drives $a$ times $20 \mathrm{~km} / \mathrm{h}$ faster on the Autobahn 
and drives all the other edges at $a v_{l i m}(e)$, one arrives at the darker route in Figure 1b). However the same route can be generated from a hierarchical routing that excludes edges with speed limits of $30 \mathrm{~km} / \mathrm{h}$ or smaller. If one uses real traffic velocities in the routing algorithm, i.e. aggregated or current data from probe vehicles for each minute of week one often finds this curve as well as the fastest route.

Apart from the risk factors described above, one might think of many more. They can be cast in weight functions of the following form:

$$
T\left(e^{\prime}, e\right)=p t(e)+t\left(e^{\prime}, e\right)+(1-p)\left[r(e)+r\left(e^{\prime}, e\right)\right] .
$$

Here $t(e)$ and $r(e)$ reflect the weights associated with edge $e$ while the weights $t\left(e^{\prime}, e\right)$ and $r\left(e^{\prime}, e\right)$ are concerned with the transition between the two edges. We distinguish further $t$ and $r$, the difference being that $t$ enters the real travel time while $r$ is a virtual risk value that only manipulates the route finding - not the travel time. The contributions to those four weights are as follows, see [9] for a different analysis:

$>t(e)$ is simply given by the edge length and the speed. The speed that enters the routing algorithm is a current speed from probe vehicles if possible. Otherwise historical or theoretical speed values are used.

$>\quad r(e)$ might not only contain a weight for number of lanes as described above but a weight for low-speed edges for example. Those edges might either be congested roads with speed limits of at least $50 \mathrm{~km} / \mathrm{h}$ or roads where $30 \mathrm{~km} / \mathrm{h}$ are allowed. In the first case there is a risk that the time to form an emergency lane becomes large. In the second case there is a risk that one has a small road that lowers the manoeuvrability of the emergency cars. Especially in pedestrian zones and play streets where the allowed velocity is $10 \mathrm{~km} / \mathrm{h}$ or less the emergency car cannot afford to drive much faster. For similar reasons as using penalties for lowspeed edges one might think of a weight for street category from small roads to German motorways. The current traffic situation (either free flow, synchronized or congested traffic) can be determined from singleprobe vehicle data by comparing the allowed and current speed and may enter with according weights. For concepts of a collective optimal route finding in congested traffic see [10]. Also roadwork contributes to the current traffic situation and has to be taken into account. A weight for architectural separation of oppositely directed lanes plays an important part especially for high densities and small number of lanes for the situation of emergency-lane formation.

$>t\left(e^{\prime}, e\right)$ : The main contribution to travel-time influencing weights for transition between edges is a penalty for turning, see above. Each time where more than a single edge follows the current edge, a delay time for turning is calculated from the angle between the two edges. For the case where more detailed information about traffic lights and their respective phases or even turning times from probe vehicles are known, they are used too. 
$>\quad r(e, e)$ concerns the following risk factors for the transition between consecutive edges: railroad crossings and other full-closure situations that cannot be resolved as well as ferry services for river crossing (as provided in individual routing) have to be displayed by the largest of weights. Beyond that we can include a penalty for the transition onto slower edges (which contain higher risk) in order for the car to stay on a strategic road. Sometimes it is necessary to have the frequent possibility of turning onto an alternative route if the current road is suddenly blocked. So an appropriate quantity to maximize is the node degree per unit length.

Finally we stress that the parameter $p$ in equation (1) interpolates between finding the fastest route (for $p=1$ ) and finding risk-minimal routes for $p=0$.

Note that while the weights $T(e)$, calculated beforehand, depend only on the current edge, the weights $T\left(e^{\prime}, e\right)$ in equation (2) depend on the previous edge as well. However the problem can be reduced to finding an optimum for $T(e)$ from the principle of node contraction $[1,2]$ : Let $e^{\prime}=(u, v)$ and $e=(v, w)$. Then those two edges can be replaced by a single edge by adding a shortcut $e^{\prime \prime}=(u, w)$. For example the travel time from $u$ over $v$ to $w$ is replaced by the sum of travel times and an appropriately scaled turning penalty, see table 2; for railroad crossings the resulting edge $e$ " will have infinite weight and so on. Those data can be preprocessed in order to enhance the performance of the algorithm; however a reasonable compromise with memory space has to be found.

\section{Risk assessment for road networks by travel time}

For the operation of critical infrastructures the German Federal Ministry of Internal Affairs [11] recommended risk assessment as an appropriate method to assess the threats. But in road-traffic management risk assessment is currently lacking. Therefore, in this chapter an evaluation function for risk in road traffic management is shown, starting at the general formula: Risk $=$ Probability of the occurrence of the threat times the expected loss in case of occurrence. To apply the risk formula for traffic management, a measure of the expected loss has to be defined. Analyzing the potential hazards and their effects in the transportation system, it comes clear that they ultimately have an impact on the travel time of the road users. All types of hazards affect the capacity of the road infrastructure (e.g. landslides) or/and the traffic demand (e.g. escape or evacuation). Both types of influence affect finally the travel time of each road user. Hence it follows, that the 'frequency of the occurrence of a threat' can be expressed as the frequency of a specific travel time. Travel times and there frequencies were measured via

floating car data (FCD) systems, also known as probe vehicle data [12-14]. Floating Cars are vehicles driving in a fleet moving with the flow of traffic, and which are equipped with a technology (e.g. GPS) to self-detect the cars' positions. The vehicles wirelessly transmit their positions and time stamps to a processing system. There, the incoming data is processed to determine traffic 
states. The FCD approach works well, if the number of equipped vehicles is large enough to ensure statistical significance of the measured traffic data [15].

Attention must be paid to the fact, that the travel time is not a loss at all, because every trip has necessarily a minimum travel time to reach the destination; only travel times beyond a limit $t_{g}$, to be defined, can be named as a loss (see Figure 2).

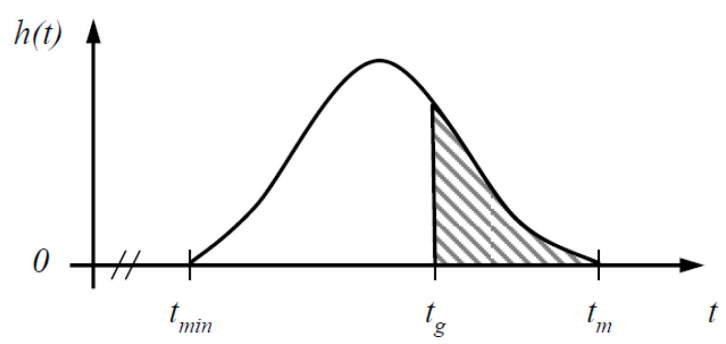

Figure 2: $\quad$ Sketch of the absolute frequency of travel times.

Therefore the risk can be calculated as follows:

$$
t_{\text {risk }}=\sum_{\text {with }_{g} \leq t \leq t_{m}} \operatorname{Prob}(t) \cdot t
$$

The formula suggests that the driver shall include a time budget of, say, 5 minutes for instance.

\section{Results}

Following, we present results derived from analysing probe-vehicle data for typical routes between a major fire station in Bonn and a destination in BonnHardtberg (cp. section 2). The data base was recorded between August 2012 and March 2013 using taxi cabs in the region of Bonn as described in section 2. For this study, the probe-vehicle data were aggregated to a 1-hour-interval.

Figure 3 displays frequencies of travel times for the route alternatives introduced in section 2. On the left side, 'cross-hatched' bars indicate the route with a minimal travel time when avoiding streets with speed limits lower than 50 $\mathrm{km} / \mathrm{h}$. The underlying grey bars refer to a route with a maximal number of lanes per kilometer. In the picture, the peak values point to the most probable travel time averaged over all working days in the respective time period.

It can be seen that the peak value of the 'cross-hatched' bars is placed at a travel time of 350 to 400 seconds whereas the peak of the grey bars has a value of above 400 seconds. This indicates that in general vehicles on the 'crosshatched' route reach the destination earlier than the cars on the 'grey' route. This appraisement may change considering the response time illustrated by the red vertical line in the figure: The histogram clearly reveals lower frequencies for the 'grey' route for travel times greater than the response time of 480 seconds. 


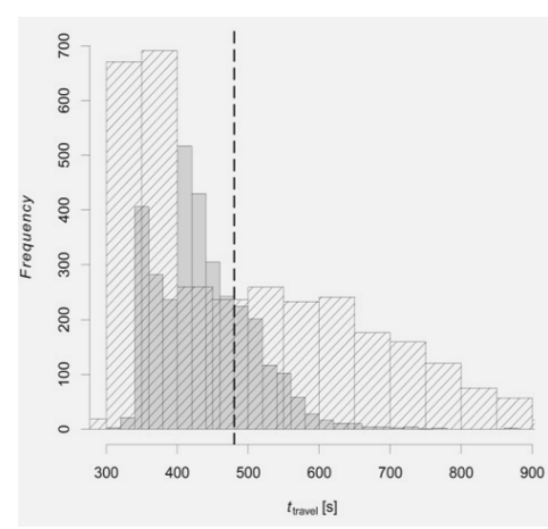

Frequencies of travel times for a route with a maximal number of lanes per kilometre (grey) and a minimal travel time when ignoring speed limits lower than $50 \mathrm{~km} / \mathrm{h}$ ('cross-hatched').

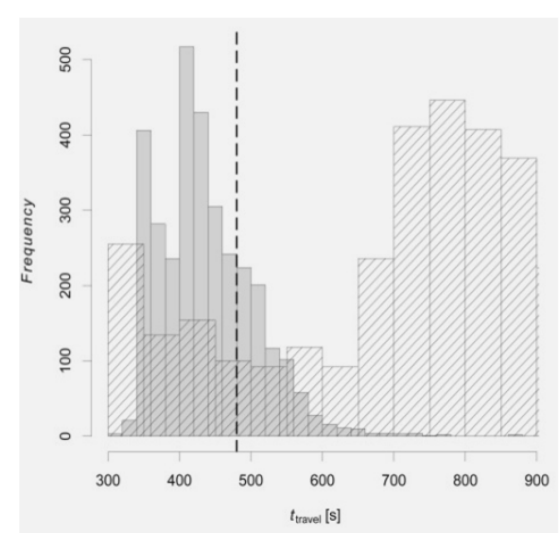

Frequencies of travel times for a route with a maximal number of lanes per kilometre (grey) and the route with the minimal length (cross-hatched').

Figure 3: Histograms of travel times over all working days for different routes from fire station 1 in Bonn to a destination in BonnHardtberg. The vertical dashed line represents the response time of 8 minutes.

In summary, vehicles are faster on the 'cross-hatched' route but also more likely not to reach their destination within the legal limit.

On the right-hand side, Figure 3 compares frequencies of travel times for the route with the minimal length ('cross-hatched') and again the route with a maximal number of lanes per kilometer (grey). The 'cross-hatched' route has a considerable amount of trips with a total travel time between 300 to 350 seconds. Nevertheless, there are a number of very high travel times in the range from 700 to 900 seconds which exceed the response time by a factor of two. In contrast, the smallest possible travel times of the 'grey' route are larger than 300 seconds. As in the figure on the left side, the number of travel times violating the legal limit is much smaller in comparison to the 'cross-hatched' route.

Table 4 illustrates these relationships in another way. It presents mean travel times well as the risks of exceeding the response time of 8 minutes defined in section 2 . The values in this table are given in seconds.

The table shows that the 'cross-hatched' route for the left side of Figure 3 has a high risk for the morning peak hour, whereas in the evening the risk is in the same range as for the 'grey' route. For the right side of Figure 3 it can be seen that despite of the possibility of realising the smallest travel times on the "crosshatched' route the mean travel times as well as the risks are always high for every period of a working day. 
Table 4: Mean and risk values of travel times for route alternatives of figure 3 of different periods of the day.

\begin{tabular}{|c|c|c|c|c|c|}
\hline Period & & \multicolumn{2}{|c|}{ Left side of Figure 3 } & \multicolumn{2}{c|}{ Right side of Figure 3 } \\
\hline & & 'cross-hatched' & 'grey' & 'cross-hatched' & 'grey' \\
\hline \multirow{2}{*}{$08: 00-09: 00$} & $\bar{t}_{\text {travel }}$ & 571.12 & 448.59 & 794.73 & 448.59 \\
\cline { 2 - 6 } & $t_{\text {risk }}$ & 434.71 & 187.01 & 664.83 & 187.01 \\
\hline \multirow{2}{*}{$18: 00-19: 00$} & $\bar{t}_{\text {travel }}$ & 468.26 & 466.04 & 819.21 & 466.04 \\
\cline { 2 - 6 } & $t_{\text {risk }}$ & 277.53 & 242.38 & 717.57 & 242.38 \\
\hline \multirow{2}{*}{24 hours } & $\bar{t}_{\text {travel }}$ & 443.02 & 426.19 & 752.20 & 426.19 \\
\cline { 2 - 6 } & $t_{\text {risk }}$ & 291.07 & 129.20 & 635.12 & 129.20 \\
\hline
\end{tabular}

This behaviour might be influenced by several facts: The route for getting minimal travel time when avoiding routes with speed limits lower than $50 \mathrm{~km} / \mathrm{h}$ ('cross-hatched' route on the left side of Figure 3) has disadvantage for the high traffic volumes of the morning peak hour. During that period higher velocities cannot be reached because of the dense traffic. In contrast, for lower traffic volumes during the day and in the evening, this route performs better in comparison to the 'grey' route.

For the right-hand side of Figure 3 it reveals that vehicles on the 'crosshatched' route with the shortest absolute length are very much influenced by the timing of the traffic lights at intersections. The very short travel times displayed in Figure 3 can only be reached for periods of weaker traffic in the night or in the early morning. For the relevant periods of the day, Table 4 shows significantly higher travel times and risk compared to the 'grey' route.

Figure 4 presents another relevant factor. Here, the standard deviations of the travel times for all working days of the sample period are plotted for the routes already presented on the right-hand side of Figure 3.

It can be seen that the travel times on the route with the minimal length (grey) are fluctuating heavily. This behaviour is caused by a high number of traffic light controlled intersections. On the one hand, a vehicle might pass these traffic lights during a 'green wave' without any delay. On the other hand, especially for periods of higher traffic volumes over the day a vehicle might be influenced at almost every intersection leading to a broad diversity of possible travel times.

In comparison to the 'grey' route, the travel times of the 'black' route (optimum between lane number average and travel time) have only very small variations. In this case, most parts of the route consist of motorways or roads of a comparable category with at least two lanes per direction. Here, the possibility of being delayed is reduced dramatically. 


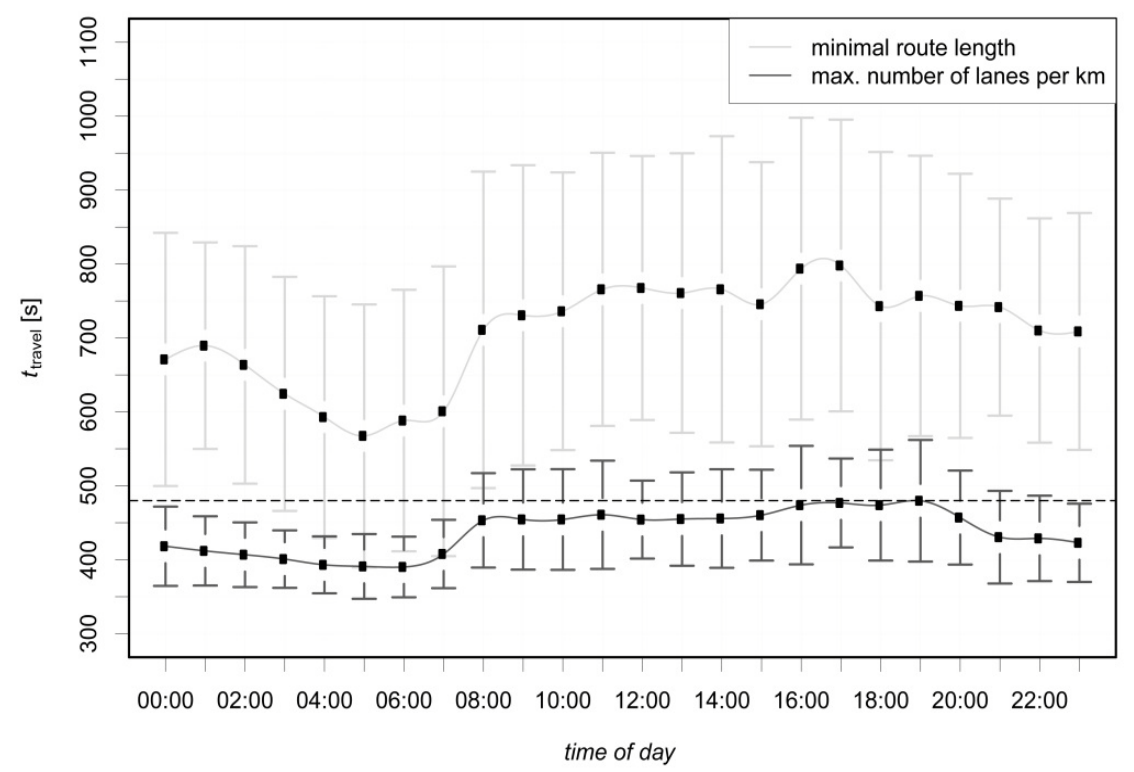

Figure 4: $\quad$ Travel times and their standard deviations for two routes from fire station 1 in Bonn to a destination in Bonn-Hardtberg. One route features an optimum between maximum number of lanes per kilometre route length and minimal travel time (black), the other route has the shortest total length (grey). The horizontal dashed line indicates the response time of 8 minutes.

\section{References}

[1] Bundesministerium des Inneren (Hrsg.): Schutz Kritischer Infrastrukturen Basisschutzkonzept Empfehlungen für Unternehmen. Berlin 2005. Download der Broschüre am 30.11.2005 von http://www.bbk.bund.de

[2] Delling, D., Sanders, P., Schultes, D., and Wagner, D., Engineering Route Planning Algorithms, in J. Lerner, D. Wagner, and K.A. Zweig (Eds.): Algorithmics, LNCS 5515, pp. 117-139, Springer-Verlag Berlin Heidelberg 2009

[3] Geisberger, R., Kobitzsch, M., and Sanders, P., Route Planning with flexible Objective Functions, in J. Lerner, D. Wagner, and K.A. Zweig (Eds.): Algorithmics, LNCS 5515, pp. 117-139, Springer-Verlag Berlin Heidelberg 2009

[4] Ngoc Nha, V.T.d, Djahel, S., and Murphy, J., A Comparative Study of Vehicles' Routing Algorithms for Route Planning in Smart Cities, VTM 2012, Satellite Workshop of IFIP Wireless Days 2012, Dublin, Ireland, November 20, 2012 
[5] Ebendt, R., and Wagner, P., An Integrative Approach to Light- and Heavy Weighted Route Planning Problems, 5th IMA Conference on Mathematics in Transport, 12.-14, London, April 2010

[6] Martins, E.Q.V., On a Multicriteria Shortest Path Problem, European Journal of Operational Research, 26(3):236-245,1984

[7] Neumann, Th., Brockfeld, E., and Sohr, A., Computing turn-dependent delay times at signalized intersections based on floating car data, Association for European Transport and contributors 2010

[8] Brockfeld, E., Neumann, Th., Sohr, A., and Kuhns, G., Turn specific vs. link based travel times calculated from floating car data' $12^{\text {th }}$ WCTR, July 11-15, Lisbon, Portugal 2010

[9] O'Har, J.P., Amekudzi, A., and Meyer, M., Risk Concepts and Applications in Transportation Asset Management: An Overview of Current Practice, Transport Research Board 91 ${ }^{\text {st }}$ Annual Meeting, 16p 2012

[10] Danila, B., Sun, Y., and Bassler, K.E. Collectively optimal routing for congested traffic limited by link capacity, Phys. Rev. E Stat. Nonlin. Soft Matter Phys. 80(6 Pt 2):066116 2009

[11] Bundesministerium des Inneren (edt.), Schutz Kritischer Infrastrukturen Risiko- und Krisenmanagement Leitfaden für Unternehmen und Behörden, Berlin 2008, Download on 2008-08-30 from www.bbk.bund.de

[12] Zheng, F.; van Zuylen, H.; Chen, Y. (2010). An investigation of urban link travel time estimation based on field sparse probe vehicle data, Proceedings of TRB 2010 89th Annual Meeting, Washington D.C. 2010

[13] Kühne, R. D.; Schäfer, R.-P.; Mieth, P.; Lorkowski, S.; Bei, X. (2005). Vehicle Probes as Data Collectors for Asian Metropolitan Areas. 4th Asia Pacific Conference, 8th - 10th Nov 2005, Xi'an, China 2005

[14] Schäfer, R. P. and Thiessenhusen, K. U. and Brockfeld, E. and Wagner, P., A traffic information system by means of real-time floating-car data, Proceedings of the 9th ITS World Congress, Chicago 2002

[15] Gössel, F.. Informationsentropische, spektrale und statistische Untersuchungen fahrzeuggenerierter Verkehrsdaten unter besonderer Berücksichtigung der Auswertung und Dimensionierung von FCDSystemen. Ph.D. Thesis, Dresden University of Technology 2005 